\title{
Extended Theoretical Considerations for Mass Resolution in the Resonance Ejection Mode of Quadrupole Ion Trap Mass Spectrometry
}

\author{
Neil S. Arnold, György Hars, ${ }^{*}$ and Henk L. C. Meuzelaar \\ Center for Micro Analysis and Reaction Chemistry, University of Utah, Salt Lake City, Utah, USA
}

\begin{abstract}
Proceeding from the pseudopotential-well approximation for ion motion in a quadrupole ion trap, mathematical expressions are derived to describe the excitation amplitude of an ion packet at a given mass-to-charge ratio. Ion-neutral collisions are incorporated to describe the damping of ion trajectories and to describe the distribution of individual ion trajectories about a mean amplitude for the ion packet. The rate of increase of the amplitude during scanning is related to expressions that describe the amplitude dispersion of the ions at the time of ejection from the trap, which is operating in a resonance ejection scanning mode to describe the temporal line width of the ejected ion packet. The temporal line width is related to mass resolution under a number of different scanning conditions. Included in the discussion are considerations of the effect on resolution of the resonance excitation voltage, temperature, pressure, noise, and buffer-gas composition. An expression for the maximum possible resolution at high ion mass-to-charge ratios is developed, and these results are compared to an existing theoretical construction. The expressions derived under the pseudopotential-well approximation are further extended to high $q_{z}$ values and compared to experimental data previously published by two other researchers. (/ Am Soc Mass Spectrom $1994,5,676-688)$
\end{abstract}

$\mathrm{R}$ ecent work on high mass, high resolution ion trap mass spectrometry has shown that threedimensional Paul-iype [1] quadrupole instruments are capable of resolution exceeding one million $[2,3]$ and detection of ions with mass-to-charge ratios greater than 50,000 [4] during operation in the resonance ejection mode [5]. Although the theory that describes the mass scanning properties of ion-trap-type instruments in both the mass-selective instability mode $[6,7]$ and in the resonance ejection modes [8] is well established, even in systems incorporating nonlinear hexapole and octapole fields [9], theory that describes the resolution characteristics for the purpose of further optimization of instrument performance has been slow to develop.

In a recent article, Goeringer et al. [10] proposed a theoretical basis for understanding high resolution mass spectra obtained in quadrupole ion traps via resonance ejection. This theory is based on two fundamental assumptions. The first assumption requires that a pseudopotential-well description of ion motion, which was extended to include ion-molecule collisions, allows the amplitude excitations of the ion to be

Address reprint requests to Dr. Neil S. Arnold, Center for Micro Analysis and Reaction Chemistry, The University of Utah, EMRL, Building 61, Room 214, Salt I ake City, UT 84112.

* On leave from the Department of Atomic Physics, Technical University of Budapest, Budapest, Hungary described by the response of a damped harmonic oscillator to an external driving frequency. The basis of this assumption is the work of Major and Dehmelt [11].

By using this assumption, the authors derived the amplitude response of an ion in the case of a linearly scanned excitation frequency. This response showed a clear dependence on scan rate and ion damping, and the width of this response line in frequency space was subsequently related to a mass line width to determine the mass resolution. The use of this frequency line width for ion excitations implies a second assumption. This assumption is that frequency dispersion of the amplitude excitation is equivalent (or at least proportional) to the spatial dispersion of the ion packet at ejection. In effect, this assumption implies that ions of a given resonant frequency are being ejected from the trap during the entire time that the excitation amplitude of the ion packet is nonzero, so that the temporal line width is the same as if the ions were not ejected at all.

In addition to not directly addressing the physics of ion packet ejection, this second assumption is troubling because it does not allow for a description of the effect of excitation amplitude on mass resolution. Furthermore, it is well known that a classical externally driven damped harmonic oscillator has no implied amplitude dispersion [12] beyond that implied by transient excitations and phase-angle differences. If scanned 
sufficiently slowly and without any other amplitude dispersion mechanism, a damped harmonic oscillator with a fixed ejection amplitude should be capable of infinite resolution.

Figure 1 clarifies this point. The wide distribution in Figure 1 describes the ion packet oscillation amplitude as a function of time, assuming the behavior of a damped harmonic oscillator. The inset distribution near the $z_{0}$ axis represents an actual distribution of ion oscillation amplitudes around the mear at the moment of ion packet ejection. In a first-order approximation, the actual temporal line width at ejection (inset near the art axis) is governed by the amplitude dispersion of the ion trajectories and the rate of amplitude increase. This line width may be considerably thinner than the temporal line width of the amplitude excitation of ions that are not ejected from the trap. In fact, if this amplitude dispersion approaches zero, the temporal line will also approach zero, corresponding to infinite resolution.

Furthermore, this second assumption is unnecessary because the same mathematical development of ionneutral momentum transfer processes used to describe the collisional damping of ion trajectories also can be used to describe the amplitude dispersion of the ion packet at the point of ejection [13]. The amplitude dispersion of the packet then may be related to the rate of increase in the ion packet oscillation amplitude at cjection to obtain temporal and mass resolution line widths. These line width expressions yield resolution descriptions that may be compared to the resolution expressions obtained from the previous frequency line width assumption and to various experimental measurements.

\section{Theory}

As illustrated in Figure 1, the temporal line width of a group of ions ejected at a given mass-to-charge ratio

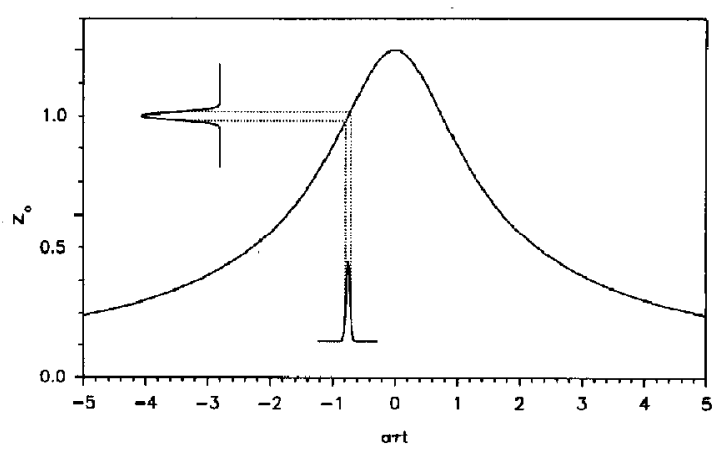

Figure 1. The relationship between ion packet excitation (full width curve), amplitude dispersion of the ion packet (inset left), and the actual temporal line width of the mass peak represented by the ion packet (inset center). may be approximated by the expression

$$
\Delta t=\frac{\Delta A}{d A / d t}
$$

Here $\Delta t$ is the temporal line width, $\Delta A$ is the amplitude dispersion at ejection, and $d A / d t$ is the rate of increase of the ion packet amplitude at ejection. By using the pseudopotential-well approximation [7, 11], we compute $d A / d t$ on the basis of considerations similar to those used by Goeringer et al. [10], whereas $\Delta A$ is derived from dispersion considerations in ion-molecule collisions.

\section{Axial Excitation of Ion Motion}

Ignoring the radial motion equations, we describe the axial motions as

$$
\frac{d^{2} z}{d t^{2}}+c \frac{d z}{d t}+\omega^{2} z=f_{\mathrm{s}} \exp \left(i \omega_{\mathrm{s}} t\right)
$$

where

$$
\begin{aligned}
\omega & =\frac{q_{z} \Omega}{2 \sqrt{2}} \\
f_{\mathrm{s}} & =\frac{e V_{\mathrm{s}}}{\sqrt{2} m r_{\mathrm{o}}} \\
q_{z} & =\frac{4 \mathrm{cV}}{m r_{\mathrm{o}}^{2} \Omega^{2}}
\end{aligned}
$$

The terms are defined as follows: $z$ is the mean axial ion position, $V$ is the fundamental $\mathrm{rf}$ drive voltage, $e$ is the ion charge, $m$ is the ion mass, $r_{0}$ is the ring electrode radius, $\Omega$ is the fundamental angular drive frequency, $V_{s}$ is the supplemental end cap excitation voltage, $\omega_{\mathrm{s}}$ is the supplemental end cap excitation frequency, and $c$ is the reduced collision frequency discussed below. The term $\omega_{\mathrm{s}}$ is the secular frequency of unforced resonant oscillations, and $f_{\mathrm{s}}$ is amplitude of external forcing normalized per unit mass.

The substitution of $z=Z(t) \exp \left(i \omega_{\mathrm{s}} t\right)$ into eq 2 along with an assumption that $Z(t)$ is a slowly varying function of time, $\omega \approx \omega_{\mathrm{s}}$, and $\omega \geqslant c$ allows us to write

$$
\frac{d Z}{d t}+\left(\frac{c}{2}+i \Delta_{\omega}\right) Z=\frac{-i f_{s}}{2 \omega}
$$

where $\Delta \omega=\omega_{\mathrm{s}}-\omega$.

It may be shown that if the secular frequency is swept linearly with time such that $\Delta \omega=$ at, where $a$ is the scan rate in radians per square second, then $Z$ can be written as

$$
\begin{aligned}
Z(x)= & \frac{-i f_{\mathrm{s}}}{2 \omega \sqrt{a}} \exp \left(\frac{-x}{\sqrt{a} \tau}-\frac{i x^{2}}{2}\right) \\
& \times \int_{-\infty}^{x} \exp \left(\frac{\xi}{\sqrt{a} \tau}+\frac{i \xi^{2}}{2}\right) d \xi
\end{aligned}
$$


where $x=a^{1 / 2} t$ and $\tau=2 / c$. The amplitude of the secular oscillation is determined by the absolute value of $Z(x)$, such that the amplitude $A=|Z(x)|$. To simplify subsequent operations, we define $F(x)$ as a dimensionless amplitude

$$
F(x)=\frac{2 \omega \sqrt{a}}{f_{6}}|Z(x)|=\frac{2 \omega \sqrt{a}}{f_{\mathrm{s}}} A(x)
$$

where $F(x)$ is given by

$$
\begin{aligned}
F(x)= & \exp \left(-\frac{x}{\sqrt{a} \tau}\right)\left[\left(\int_{\infty}^{x} \exp \left(\frac{\xi}{\sqrt{a} \tau}\right) \cos \left(\frac{\xi^{2}}{2}\right) d \xi\right)^{2}\right. \\
& \left.+\left(\int_{\infty}^{x} \exp \left(\frac{\xi}{\sqrt{a} \tau}\right) \sin \left(\frac{\xi^{2}}{2}\right) d \xi\right)^{2}\right]^{1 / 2}
\end{aligned}
$$

Direct evaluation of these integrals is difficult , and closed-form solutions do not exist, but as shown in refs 10 and 11 , it is possible to utilize approximations for the two cases where $a^{1 / 2} \tau=1$ and $a^{1 / 2} \tau \leqslant 1$. These integrals also may be evaluated numerically in regions where these simple approximations are not available.

For $a^{1 / 2} \tau \& 1$, we utilize a result obtained by integration by parts, so that

$$
F(x)=\frac{\sqrt{a} \tau}{\sqrt{1+a \tau^{2} x^{2}}}
$$

gives the excitation amplitude. This expression may be differentiated with respect to time to obtain

$$
\frac{d F}{d x}=\frac{(\sqrt{a} \tau)^{3} x}{\left(1+a \tau^{2} x^{2}\right)^{3 / 2}}
$$

This latter equation describes the rate of increase of the ion envelope near ejection.

For the case of $a^{1 / 2} \tau \gg 1$ we require an approximation that describes the rising side of the amplitude envelope so that the slope in the vicinity of resonant frequency at $x=0$ may be determined. In this limit, we can rewrite eq 6 in terms of Fresnel integrals [14], so that

$$
\begin{gathered}
F(x)=\sqrt{\pi} \sqrt{\left(\frac{1}{2}+C\left(\frac{x}{\sqrt{\pi}}\right)\right)^{2}+\left(\frac{1}{2}+S\left(\frac{x}{\sqrt{\pi}}\right)\right)^{2}} \\
C(x)=\int_{0}^{x} \cos \left(\frac{\pi \xi^{2}}{2}\right) d \xi \\
S(x) \equiv \int_{0}^{x} \sin \left(\frac{\pi \xi^{2}}{2}\right) d \xi
\end{gathered}
$$

shows a square root dependence of the diffraction intensity pattern produced by an infinite straight edge in wave optics [15]. Subsequent differentiation with respect to $x$ gives

$$
=\frac{\frac{d F}{d x}}{\sqrt{\left.\left(\frac{1}{2}+C\left(\frac{x}{\sqrt{\pi}}\right)\right) \cos \left(\frac{x^{2}}{2}\right)+\left(\frac{1}{2}+S\left(\frac{x}{\sqrt{\pi}}\right)\right) \sin \left(\frac{x^{2}}{2}\right)\right)^{2}+\left(\frac{1}{2}+S\left(\frac{x}{\sqrt{\pi}}\right)\right)^{2}}}
$$

\section{Amplitude Dispersion}

The average rate of momentum loss due to collisions is related to the reduced collision frequency $c$. A mathematical derivation for $c$ is given by

$$
c=\left(\frac{M}{m+M}\right) n v_{\mathrm{r}} \sigma_{D}
$$

where $m$ is the ion mass, $M$ is the mass of colliding neutrals, $n$ is the number density of neutrals, $v_{r}$ is the average relative speed of the colliding ion and neutral, and $\sigma_{\nu}$ is the collision cross section, and proceeds from momentum transfer arguments that are outlined elsewhere [13], wherein the ion is assumed to be "drifting" in a constant electric field. The present developments assume that although the ions undergo accelerated motion in the ion trap oscillations, the average momentum damping is still the same.

The same arguments that allow the development of the reduced collision frequency expressions also may be applied to velocity dispersion of the ion packet [13] caused by collisions with neutrals. This dispersion is based on the discontinuous nature of the ion damping. Following Mason and McDaniel [13] and considering only the axial excitations of the ions, we write the relative velocity dispersion as for ions traveling at drift velocity $v_{\mathrm{d}}$

$$
\left(\Delta V_{z}\right)^{2}=\overline{v_{z}^{2}}-v_{\mathrm{d}}^{2}=\frac{k T}{m}+\frac{C^{*}}{3} \frac{4 m+M}{2 m+M} \frac{M}{m} v_{\mathrm{d}}^{2}
$$

where $\left(\Delta V_{z}\right)^{2}$ is the velocity dispersion about the mean $v_{\mathrm{d}}$ in the $z$ direction, $v_{z}^{2}$ is the thermal average of the square of ion velocity in the $z$ direction, $k$ is the Boltzmann constant, $T$ is absolute temperature, and $C^{*}$ is a constant that has a value on the order of 1 depending on the $m / M$ ratio and the nature of ionneutral scattering.

As mentioned before, eqs 11 and 12 are both developed for the case of constant electric field. Use of eq 11 in the present case requires the assumption that the overall ion packet is in dynamic equilibrium with the 
neutrals via collisions, so that $c$ may represent the average damping of the ion packet. Equation 12 represents the fluctuations around this equilibrium, and the earlier assumption that $\omega>c$ implies that the velocity dispersion may be averaged over time by using the root mean square (rms) value of $v_{\mathrm{d}}$. Further, because that particle is undergoing simple harmonic motion, the expression $v_{z}=\omega z$ allows us to relate the velocity dispersion to the amplitude dispersion of the ion packet.

The amplitude dispersion of the ion packet in dynamical equilibrium with the surrounding neutrals may therefore be represented as

$$
\Delta A=\frac{1}{\omega_{s}} \sqrt{\frac{k T}{m}+\frac{C^{*}}{3} \frac{4 m+M}{2 m+M} \frac{M}{m} v_{\mathrm{rms}}^{2}}
$$

where $v_{\text {rms }}$ is the rms velocity amplitude due to the external driving field. At the point of ejection, $A_{\text {ej }}=$ $r_{\mathrm{o}} / 2^{1 / 2}, v_{\mathrm{rms}}=\omega_{\mathrm{s}} r_{\mathrm{o}} / 2$ and substitution into eq 13 gives

$$
\Delta A=\sqrt{\frac{k T}{\omega_{\mathrm{s}}^{2} m}+\frac{C^{*}}{12} \frac{4 m+M}{2 m+M} \frac{M}{m} r_{o}^{2}}
$$

This expression indicates that the amplitude dispersion at ion ejection is directly related to the effective ion temperature in the axial direction [13]. Additional consideration of velocity dispersion in the radial direction would allow $m(\Delta V)^{2}$ (similar to eq 12) to be related to the effective ion temperature as discussed in Mason and McDaniel [13]. This fact has additional implications for instrument resolution and spectral quality, which may be degraded by collision-induced dissuciation of ions caused by scanning of the lons from the trap.

\section{Minimum Ejection Line Width}

Direct substitution into eq 1 of eq 14 along with eq 8 or 10 yields an expression for temporal line width $\Delta t$ depending on the actual time of ion packet ejection $t_{\mathrm{e} \text { - }}$ Although this substitution may be performed at any value, the primary interest is in the maximum obtainable resolution at a given scan speed that will occur at the minimum of $\Delta t$ or the maximum of $d A / d t$.

At the point in time $x_{\mathrm{ej}}\left(=a^{1 / 2} t_{\mathrm{ej}}\right)$ where the ion package is ejected, the amplitude of oscillation is given by $A\left(x_{\mathrm{ej}}\right)=r_{\mathrm{o}} / 2^{1 / 2}$. Based on eq 5 , this ejection point is determined by the excitation amplitude $f_{\mathrm{s}}$, so that

$$
f_{\mathrm{s}}=\frac{\sqrt{2} \omega_{\mathrm{s}} r_{\mathrm{o}} \sqrt{a}}{F\left(x_{\mathrm{ej}}\right)}
$$

describes the excitation required to eject the ion packet at time $x_{\mathrm{ej}}$.

To evaluate the maximum of $d A / d t$, eq 5 is differentiated with respect to $t$ whereas eq 15 is substituted for $f_{\mathrm{s}}$ to obtain

$$
\frac{d A}{d t}=\frac{r_{\mathrm{o}} \sqrt{a}}{\sqrt{2} F\left(x_{\mathrm{ej}}\right)} \frac{d F\left(x_{\mathrm{ej}}\right)}{d x}=\frac{r_{\mathrm{o}} \sqrt{a}}{\sqrt{2}} \frac{d \ln F\left(x_{\mathrm{ej}}\right)}{d x}
$$

$d A / d t$ is proportional to the ratio of $d F / d x$ to $F$. This ratio, also expressed as $d \ln F / d x$, is plotted for various values of $a^{1 / 2} \tau$ in Figure 2. Two different scalings are provided in this figure because the limiting case $a^{1 / 2} \tau \notin 1$ has a natural scaling of $a \tau t$, as seen in eqs 7 and 8 , whereas the case $a^{1 / 2} \tau>1$ scales to $a^{1 / 2} t$, as seen in eqs 9 and 10. At a given scan speed and damping, the maximum resolution depends on maximizing $d \ln F / d x$. This maximum occurs at $t_{\mathrm{m}}=$ $-1 / a \tau$, as illustrated in Figure $2 a$, for all scan-rate and damping values! This result establishes the temporal shift of the ion ejection point (obtained at maximum resolution) relative to the resonant frequency of ion oscillations.

The maximum of $d A / d t$ at ion ejection as a function of $a$ and $\tau$ may be obtained for the two limiting cases on the basis of a similar evaluation as was performed to obtain eqs 8 and 10. For $a^{1 / 2} \tau+1$, the rate of
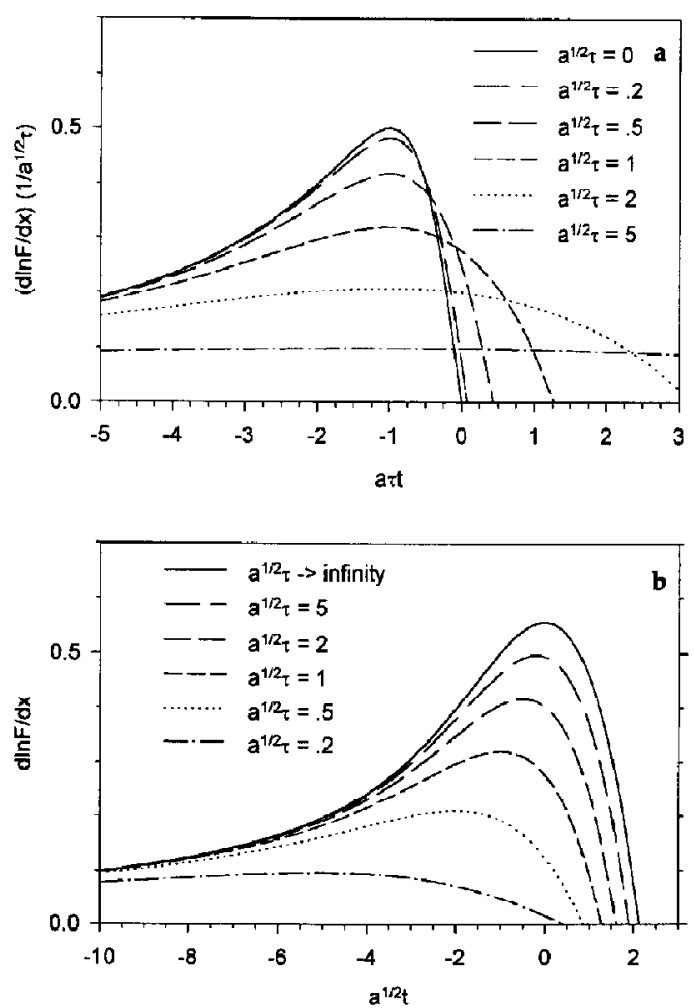

Figure 2. Derivative of the $\log$ of $F(x)$ for various scan-rate and damping factors as a function of art and $a^{1 / 2} \tau$. All curves share a maximum at art $=-1$. The maximum on each curve indicates the optimum excitation amplitude and resolution. 
maximum amplitude increase is given by

$$
\left.\frac{d A}{d t}\right|_{\max }=\frac{r_{\mathrm{o}} a \tau}{2 \sqrt{2}}
$$

whereas for $a^{1 / 2} \tau \gg 1$, the rate of maximum amplitude increase is given by

$$
\left.\frac{d A}{d t}\right|_{\max }=\frac{r_{\mathrm{o}} \sqrt{a}}{\sqrt{2 \pi}}
$$

As previously indicated, eq 1 provides an expression for temporal line width of a mass peak based on the amplitude dispersion and the rate of increase of the amplitude of ion motion. If eq 14, along with eq 17 or 18 , is substituted into eq 1 to give the minimum line width at fixed scan-rate and damping conditions, the temporal line width is given by

$$
\Delta t=\frac{2 \sqrt{2}}{a \tau} \sqrt{\frac{k T}{m r_{\mathrm{o}}^{2} \omega_{\mathrm{s}}^{2}}+\frac{C^{*}}{12} \frac{4 m+M}{2 m+M} \frac{M}{m}} \quad \sqrt{a} \tau \leqslant 1
$$

and

$\Delta t=\sqrt{\frac{2 \pi}{a}} \sqrt{\frac{k T}{m r_{0}^{2} \omega_{s}^{2}}+\frac{C^{*}}{12} \frac{4 m+M}{2 m+M} \frac{M}{m}} \quad \sqrt{a} \tau \gg 1$

\section{Results and Discussion}

\section{Mass Resolution}

The work of Goeringer et al. [10] established the relationship between temporal line width and mass resolution as

$$
\frac{m}{\Delta m}=\frac{\omega}{\Delta \omega}=\frac{\omega}{\Delta t}
$$

(Note that the subscript " $s$ " has been dropped from this and subsequent secular frequency $\omega$ references.) This expression, along with those in eqs 19 and 20, allows the evaluation of mass resolution, but a necessary condition for the use of eq 1 in the derivation of these equations is that $\Delta A$ must be small relative to $r_{\mathrm{i}} / 2^{1 / 2}$ at ejection. In practice, this condition is equivalent to $m / M>1$ and is readily met by the use of a light buffer gas during mass analysis. Additionally, the derivation of eqs 8 and 10 requires that $\omega$ be near resonance at ejection. This condition is met by the use of maximum $d A / d t$ values for the two cases.
By using $m / M>1$, we obtain eqs 22 and 23 for the two regimes:

$$
\begin{array}{cc}
\frac{m}{\Delta m}=\frac{\omega}{\sqrt{8} \sqrt{\frac{k T}{m r_{0}^{2} \omega^{2}}+\frac{C^{*}}{6} \frac{M}{m}}} & \sqrt{a} \tau \leqslant 1 \\
\frac{m}{\Delta m}=\frac{\omega}{\sqrt{2 \pi a} \sqrt{\frac{k T}{m r_{0}^{2} \omega^{2}}+\frac{C^{*}}{6} \frac{M}{m}}} \quad \sqrt{a} \tau>1
\end{array}
$$

These two expressions may be used directly to obtain the limiting behavior of the resolution in the high and low scan speed cases. Lower and upper bounds for the maximum obtainable resolution can be determined from the limiting behavior, and two such bounds are given by

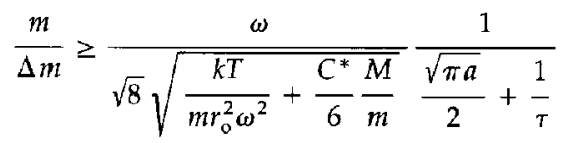

and

$$
\frac{m}{\Delta m} \leq \frac{\omega}{\sqrt{8} \sqrt{\frac{k T}{m r_{0}^{2} \omega^{2}}+\frac{C^{*}}{6} \frac{M}{m}}} \frac{1}{\sqrt{\frac{\pi a}{4}+\frac{1}{\tau^{2}}}}
$$

These last four equations describe for various limits the maximum obtainable resolution at a given scan speed and damping. Figure 3 illustrates the actual scan-rate dependence of the mass resolution as a function of $a^{1 / 2} \tau$. This scan-rate dependence is based on numerical integration of $d \ln F / d x$ at $t=-1 / a \tau$. Also included in the figure are the bounds provided by eqs 24 and 25 , which are established by the limiting behavior of the resolution.

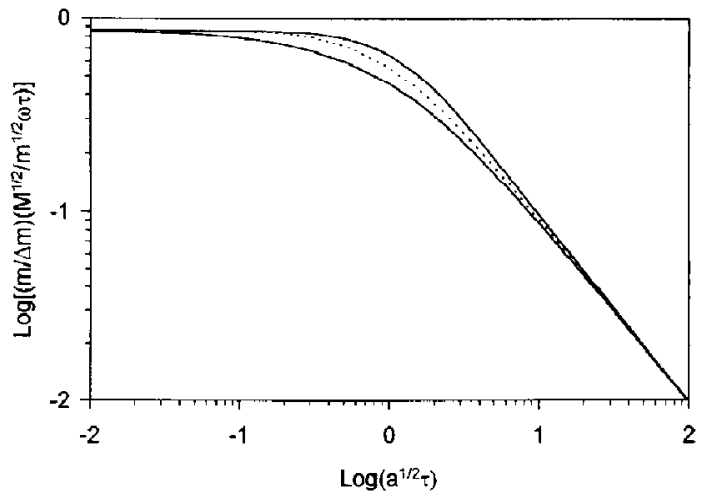

Figure 3. Resolution as a function of the scan rate and damping factor $a^{1 / 2} \tau$ in the limiting case $k T \rightarrow 0$ and $C^{*}=1$. Dotted line is based on numerical integration, whereas solid lines are based on the bounds presented in eqs 23 and 24 . 


\section{Mass Dependence of Instrument Resolution}

Equations 24 and 25 describe the mass dependence of the maximum obtainable resolution, but in fact both $a$ and $\tau$ have mass dependence that should be made explicit. This affects both the resolution expressions of eqs 22 through 25 as well as appropriate axial excitation amplitudes discussed subsequently in eqs 37 through 40.

The mass dependence of $a$ is straightforward: $a$ is directly related to the mass scan rate via the differential form of eq 21 as seen in

$$
a=\frac{d \omega}{d t}=\frac{e \omega}{m} \frac{d(m / e)}{d t}
$$

Although the frequency scan rate for a given mass-tocharge ratio remains constant over the course of the scan, the effective (frequency) scan rate decreases with increasing mass. This important result may be coupled immediately to eq 23 (high scan speed limit) to obtain

$$
\begin{aligned}
\frac{m}{\Delta m}= & \left(m / 2 \sqrt{\frac{k T}{r_{\mathrm{o}}^{2} \omega^{2}}+\frac{C^{*} M}{6}}\right) \sqrt{2 \omega / e \pi \frac{d(m / e)}{d t}} \\
\sqrt{a} \tau & \approx 1
\end{aligned}
$$

This result, where resolution is directly proportional to mass, may be used to illustrate clearly why unit mass resolution is readily maintained over the entire scan range of the "classic" ion trap [16, 17] system.

A brief qualitative argument illustrates this fact. It is well recognized that an ion trap that uses axial modulation [8] qualitatively gives resolution behavior similar to the classic mass-selective instability scan, whereas various researchers $[10,18,19]$ have noted that line width behavior at higher $q_{z}$ values (i.e., $q_{z}>0.4$ ) should yield qualitatively similar characteristics. At the high $q_{z}$ values (near $q_{z}=0.9$ ) that are used for axial modulation, $k T<C^{*} M r_{o}^{2} \omega^{2} / 6$, so that in classic mass-selective instability at high scan speeds, eq 28 pertains:

$$
\frac{m}{\Delta m} \propto m / \sqrt{e M \frac{d(m / e)}{d t}} \quad \sqrt{a} \tau \geqslant 1, k T \Leftrightarrow \frac{C^{*}}{6} M r_{0}^{2} \omega^{2}
$$

The ion-mass $m$ and neutral-molecule buffer-mass $M$ [20] dependence of this expression are in good agreement with the known behavior of the classic mass-selective instability scan. The scan-rate dependence also agrees with high scan speed behavior described by Louris et al. [19] (see Figure 6a). This result further implies that resolution is independent of pressure if ions are sufficiently cooled prior to spectrum scanning $[6,7,21]$.
Like $a, \tau$ also has mass dependence because $\tau=2 / c$ and $c$ is defined in eq 11. In addition to the explicit terms, $v_{\mathrm{r}}$ and $\sigma_{D}$ may also carry some mass dependence. The special case utilized by Goeringer et al. [10] involved an induced dipole-interaction model between the ion and the neutral molecules, because for this model, the collision frequency is truly independent of ion velocity in $v_{\mathrm{r}}$. In such a case (by using $m>M$ ) we obtain

$$
c=\frac{2 \pi n e}{m} \sqrt{\frac{\alpha_{\mathrm{p}} M}{\varepsilon_{\mathrm{o}}}}
$$

where $\alpha_{\mathrm{p}}$ is the polarizability of the neutral and $\varepsilon_{\mathrm{o}}$ is the permittivity of free space.

The velocity independence of $c$ is essential to the solution of eq 2 as presented in eqs 7 through 10 . In this ion-induced dipole-interaction model, this velocity independence is preserved, although as seen in eq 11 , the reduced collision frequency $c$ in general depends on the relative velocity between the ion and a colliding neutral molecule. However, even in a more general ion-neutral interaction model, the relative velocity $v_{r}$ (and therefore c) is still velocity independent if $k T \Rightarrow$ $C^{*} M r_{0}^{2} \omega^{2} / 6$. This condition is the same as that which will be discussed subsequently as a temperaturelimited resolution regime (see eq 32).

Nevertheless, this expression has some general applicability for lower mass ions, and utilizing eq 29 , one may rewrite eq 22 as

$$
\frac{m}{\Delta m} \simeq \frac{\sqrt{\varepsilon_{\mathrm{o}}} \omega m^{3 / 2}}{\pi n e \sqrt{8 a_{\mathrm{p}} M} \sqrt{k T / r_{\mathrm{o}}^{2} \omega^{2}+\mathcal{C}^{*} M / 6}} \quad \sqrt{a} \tau \nLeftarrow 1
$$

This is the low scan speed analog of eq 27.

It must be noted that eqs 27,28 , and 30 all show a difference in resolution between ions of the same mass-to-charge ratio when these ions have different charge states. More explicitly, each equation can be written as a function of $m / e$ multiplied by $m^{1 / 2}$. In this situation, the improvement in resolution for a multiply charged ion at a given $m / e$ value is proportional to the number of elemental charges. It is important to note that this implies that under a fixed set of scanning conditions, the maximum mass resolution will be obtained for the singly charged ion.

The primary means of delineating the two operating regimes has been to consider the value of $a^{1 / 2} \tau$ relative to 1 . This value also has an explicit mass dependence, based on eqs 26 and 29, as is given by

$$
\sqrt{a} \tau=\frac{1}{\pi n} \sqrt{\left(\varepsilon_{\mathrm{o}} \omega_{m} \frac{d(m / e)}{d t}\right) / \alpha_{\mathrm{p}} M e}
$$

It is clear that the primary dependence in this expression is pressure, whereas all other factors are ex- 
pressed as the square root. Nevertheless, under full scan experiments, the mass dependence does provide for differences of a factor of 3 or 4 for different ions in a given scan, yielding distinctive resolution behavior.

\section{Temperature and Collisional Focusing}

The effect of temperature on resolution arises from the ion packet dispersion term in the denominator of eqs 22 through 25 . The dependence of resolution on temperature is governed by the relative values of the temperature itself and the frequency of supplemental excitation. The critical point occurs when

$$
k T=\frac{1}{3} M \bar{V}_{\mathrm{M}}^{2}=\frac{C^{*}}{6} M r_{\mathrm{o}}^{2} \omega^{2}
$$

where $V_{M}$ is the mean thermal velocity of the neutrals. This point occurs when the average axial ion velocity at ejection $\left(v_{\mathrm{rms}}=r_{0} \omega / 2\right)$ exceeds the mean thermal velocity of the neutrals. For ejection frequencies $\left(q_{z}\right.$ values) below this critical point, the resolution is limited by the dispersion of the "stationary" ion packet before excitation, which is a function of system temperature and may be represented as

$$
\frac{m}{\Delta m} \geq \frac{\sqrt{m} r_{\mathrm{o}} \omega^{2}}{16 \sqrt{2 k T}} \frac{1}{\sqrt{\pi a} / 2+1 / \tau} \quad k T>\frac{C^{*}}{6} M r_{0}^{2} \omega^{2}
$$

This expression clarifies that increases in ejection frequency greatly enhance the resolution until the condition of eq 32 is met. In a standard commercial ion trap mass spectrometry (ITMS) instrument ( $r_{\mathrm{o}}=1 \mathrm{~cm}, \Omega=$ $\left.6.911 \times 10^{6} \mathrm{~s}^{-1}\right)$ at $0^{\circ} \mathrm{C}$, the condition of eq 32 is computed to occur at a supplemental frequency of 29.3 $\mathrm{kHz}\left(q_{z}=0.075\right)$ for helium buffer gas and $11.1 \mathrm{kHz}$ $\left(q_{z}=0.028\right)$ for nitrogen (assuming $C^{*}=1$ ). Above these values, the increase in resolution with increasing frequency will be a modified linear relationship described by

$$
\frac{m}{\Delta m} \geq \frac{(\omega / \sqrt{8}) \sqrt{6 m / C^{*} M}}{\sqrt{\pi a} / 2+1 / \tau} \quad k T \ll \frac{C^{*}}{6} M r_{0}^{2} \omega^{2}
$$

This is, of course, the opposite limiting case from eq 33. In this case, we see that buffer molecular mass becomes an important limiting factor in higher ejection frequency cases. In either case, increasing the ion mass or the excitation frequency yields increased resolution.

Figure 4 illustrates the relationship between temperature, buffer mass, and excitation frequency for five different buffer gases in both low and high scan speed limits. At low temperatures (the buffer-mass-limited case of eq. 28), the condition of maximum resolution is obtained, whereas increasing temperature provides a degraded resolution although the inflection point may
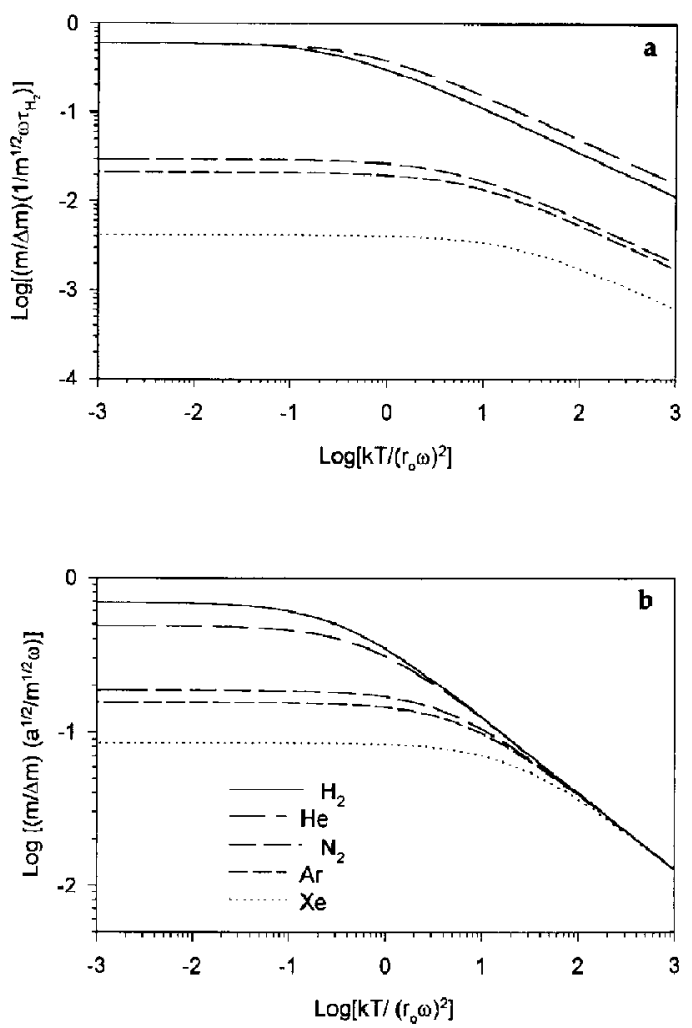

Figure 4. Temperature dependence of resolution for various buffer gases: (a) high scan speed limit $a^{1 / 2} \tau>1$; (b) low scan speed limit $a^{1 / 2} \tau<1$.

be varied by adjustment of the excitation frequency. One interesting feature of the low scan speed limit is the fact that helium rather than hydrogen provides the highest resolution. This is primarily due to the significantly lower polarizability (by more than a factor of 2) of helium.

The temperature of neutral buffer molecules has been used in the development of amplitude dispersion equations 12 through 14. This approach is consistent with the pseudopotential-well model developments of Major and Dehmelt [11]. It is clear that the actual ion temperatures reported in the literature [21-23], which are typically higher than the buffer ion temperature, depend also on the effective ion temperatures produced by non-thermal excitations of the ion trajectories. Several means for these excitations have been discussed including nonlinear resonances $[9,24]$, drive frequency harmonics [24], parasitic electrical signals, mechanical vibrations, and electrical noise [25].

Although these excitation sources must be recognized for their temperature-related effects in any ion chemistry experiment, it is clear that the hottest point for ions in virtually all ion trap experiments occurs at ion ejection. At ejection, the amplitude dispersion described by eq 14 has a direct thermal component and 
an axial excitation component. The axial excitation component of this dispersion is proportional to the product $A \omega$, as was described in the derivation of eq 14, for each frequency component. As long as the $A \omega$ product for other non-secular oscillation frequencies is small with respect to $\tau_{\mathrm{o}} \omega_{\mathrm{s}} / 2^{1 / 2}$, the dispersion factors for these other oscillations may be neglected in the spatial amplitude dispersion term.

\section{Excitation Amplitude and Mass Shift}

During the development of minimum ejection line width expressions, eq 15 described the relationship between the ejection point of the ion packet and the supplementary excitation amplitude. Further it was observed that $d A / d t$ has a clear maximum for given $a$ and $\tau$ values when the ejection point occurs at $\Delta t_{m}$ as given by

$$
\Delta t_{\mathrm{m}}=-\frac{1}{a \tau}
$$

(see Figure 2). This time is measured relative to the resonance point in the scan (e.g., $t=0$ occurs at $\omega=$ $\omega_{\mathrm{s}}$ ). Substituting eqs 26 and 29 into this expression gives eq 36 , which illustrates the effect of pressure, scan rate $(d(m / e) / d t)$, and ejection frequency on the ejection time at maximum resolution:

$$
\Delta t_{\mathrm{m}}=-\left(\pi n /\left(\omega \frac{d(m / e)}{d t}\right)\right) \sqrt{\frac{\alpha_{p} M}{\varepsilon_{0}}}
$$

This ejection time implies a mass shift that can be determined based on eq 21 to offer a comparison between mass shift and mass resolution. The relative mass shift at maximum resolution is given by

$$
\frac{m}{\Delta m}=-\omega \tau=-\frac{\omega m}{\pi n e} \sqrt{\frac{\epsilon_{\mathrm{o}}}{\alpha_{\mathrm{p}} M}}
$$

which is independent of scan rate, but clearly dependent on ion mass-to-charge ratio, buffer mass, and pressure. For low scan speeds $\left(a^{1 / 2} \tau \leqslant 1\right)$ this value may be directly compared to those in eqs 22 and 30 .

This result is somewhat limited in that it only applies to the mass shift after the excitation amplitude $f_{\mathrm{s}}$ is adjusted to obtain maximum resolution. If the amplitude of the secular drive is not adjusted to yield maximum resolution for the ion mass of interest, it is clear that eq 15 will govern the mass shifts. The maximum delay possible in an ion ejection system occurs at the maximum value of amplitude envelope. In Figure 2 this corresponds to the point where $d \ln F / d x=0$. At scan-rate extremes, ejection can only be delayed for a small time after resonance. For $a^{1 / 2} \tau+1$, the maximum delay occurs approximately at $t=+2 \tau$, whereas for $a^{1 / 2} \tau \Rightarrow 1$, the maximum delay occurs at about $t=+2 / a^{1 / 2}$. By overdriving the axial excitation am- plitude, ions may be ejected at any time prior to resonance, so that the minimum ejection time $t$ is not limited by any boundary.

Because maximum resolution occurs at $x_{\mathrm{m}}=$ $-1 /\left(a^{1 / 2} \tau\right)$, as seen in Figure 2, substitution of this value into eq 15 provides an expression for optimum excitation amplitude. Combined with the definition of $f_{\mathrm{s}}$ in eq $2, f_{\mathrm{s}}$ can be directly related to the excitation voltage amplitude, so that

$$
V_{\mathrm{s}}=\frac{\sqrt{2} m r_{\mathrm{o}} f_{\mathrm{s}}}{e}=\frac{2 \omega m r_{\mathrm{o}}^{2} \sqrt{a}}{F(-1 / \sqrt{a} \tau)}
$$

The behavior of $V_{s}$ as a function of $a^{1 / 2} \tau$ is the inverse of the behavior of resolution in Figure 3. The value of $V_{\mathrm{s}}$ may be approximated in a manner similar to that for $m / \Delta m$ in eqs 24 and 25. The limiting cases arc given by the expressions

$$
V_{\mathrm{s}} \geq \frac{2 \sqrt{2} \omega m r_{\mathrm{o}}^{2}}{e} \sqrt{\frac{1}{\tau^{2}}+\frac{a}{\pi}}
$$

and

$$
V_{\mathrm{s}} \leq \frac{2 \sqrt{2} \omega m r_{0}^{2}}{e}\left(\frac{1}{\tau}+\frac{\sqrt{a}}{\sqrt{\pi}}\right)
$$

These expressions may again he substituted with eqs 26 and 29 to illustrate the mass dependencies at maximum resolution conditions. Further, substituting eq 26 for the case $a^{1 / 2} \tau=1$ yields

$$
V_{\mathrm{s}} \simeq 2 \sqrt{2} \omega r_{\circ}^{2} \sqrt{\frac{m \omega}{\pi e} \frac{d(m / e)}{d t}} \quad \sqrt{a} \tau>1
$$

whereas substituting eq 29 in the case $a^{1 / 2} \tau \leftarrow 1$ yields

$$
V_{\mathrm{s}}=2 \sqrt{2} \omega \pi n r_{\mathrm{o}}^{2} \sqrt{\frac{\alpha_{\mathrm{p}} M}{\varepsilon_{\mathrm{o}}}} \quad \sqrt{a} \tau \leqslant 1
$$

Of particular interest is the mass dependence of both expressions, which indicate that excitation frequency amplitudes may benefit from scanning in a manner similar to the if scanning of the instrument. It should be noled that eq 41 describes a beliavior that is qualitatively similar to that of a classical ITMS system for adjustment of the excitation amplitude relative to the scan rate and ion mass variables [26] and agrees well with ref 19 at $4.2 \mathrm{~V}$.

This analysis is based on the assumption that a simple dipole field describes the electric field produced by the supplemental end cap voltages. Actual field values vary considerably from this dipole ideal. Even near the origin, correction values for the axial excitation electric field have been reported as 0.8 [27] and 0.877 [28] based on experimental and theoretical considerations, respectively. These dipole field correc- 
tion values are a function of both radial and axial positions, and they are expected to be considerably smaller with radial displacements near the ring and to increase to unity near the center of the end caps.

\section{Limitations and Comparison to Existing Theory}

The present theoretical construction is an important departure from previous theoretical work [10] for three reasons. First, the high scan speed behavior is not dependent on pressure and proceeds as $a^{1 / 2}$ rather than $a \tau$ as in the previous analysis. It should be noted that this result is consistent with a large body of data that show resolution to be constant over a broad range of pressures when sufficient time is allowed to thermalize the ions before scanning $[7,22]$.

Second, the present theory predicts, via eqs 39 through 42 , the excitation voltages required to obtain maximum resolution at any scan speed. However, we reiterate that important nonlinear field effects were disregarded in the derivation of these two equations. A correction factor is necessary to predict required excitation voltages accurately for a given resolution; one correction factor is probably obtained best from experiment, but the scan-rate dependencies and temporal line shifts should not be altered by these considerations.

Third, the ion dispersion description implies that resolutions are directly dependent on the relative masses of the buffer neutrals and the ions themselves owing to collisional focusing. Furthermore, this effect is independent of the scan-rate regime. This effect describes how higher resolutions are produced for higher masses in any continuous scan. This term also incorporates the effects of temperature (and, qualitatively, electrical noise) into the consideration of resolution.

Figure 5 illustrates a predicted resolution comparison between the present result and the previous frequency line width mode [10]. The resolution predicted in the present theory is actually higher in both scan-rate regimes. In the slow scan regime the resolution is higher owing to the collisional focusing of the higher mass ions by the light neutrals. This focusing effect is also present at high scan speeds, but it is more important to note that the $1 / a^{1 / 2}$ behavior of the resolution implies higher resolution even without this focusing effect.

Note that several important sources of amplitude dispersion have been neglected in the present theory to focus on the ion behavior in a damped harmonic oscillator model. During the previous discussion of temperature, electrical noise was dismissed as a major source of spatial ion packet dispersion, but temporal and frequency dispersion effects, which could result from noise in the radiofrequency drive or other electrical and mechanical sources, were not considered. It is worth noting that $\Delta \omega_{\mathrm{s}}$ is directly proportional to $\Delta V$ in the drive voltage and that this may yield significant
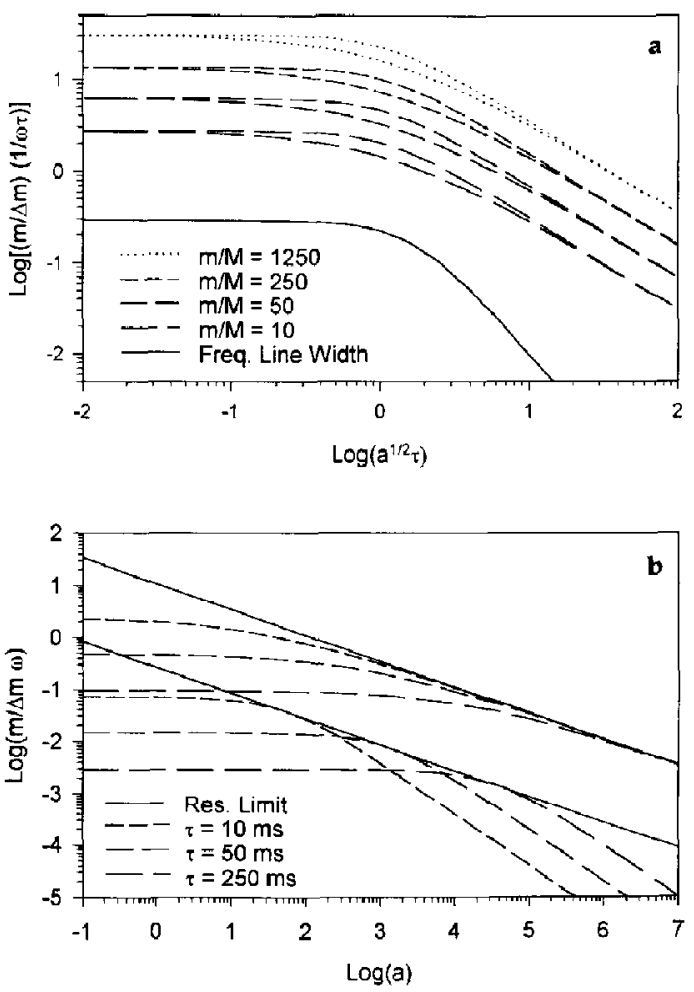

Figure 5. (a) Comparison of mass resolution al four different $m / M$ values in the low temperature limit. Both upper and lower bounds are shown. The behavior of the previous frequency line with model is also shown for comparison. (b) Sperific comparison of the present model at $m / M=125$ with the existing frequency line width model. The upper four broken curves illustrate the present model at three damping factors, whereas the lower 3 illustrate the frequency line width model of the same three values. The two solid lines indicate the maximum obtainable resolution as a function of scan rate for the two models.

temporal fluctuations in the amplitude of the ion packet during scanning.

Geometrical considerations also have been neglected. In a standard commercial instrument, the axial position that corresponds to the ion exit aperhure is a function of radius. If the exit aperture is only $3.5 \mathrm{~mm}$ in radius, the $\Delta A$ of the aperture may be as large as $0.04 r_{0}$, which is the same as collision-based dispersion produced by an $m / M$ ratio of 92 (e.g., $m / z 370$ in He buffer) in the low temperature limit. Clearly for higher masses the aperture size could easily be the resolution-limiting element.

Geometrical variations already mentioned with respect to the supplemental axial excitation field will also reduce resolution because the effective excitation voltage is a function of radius in the trap. This second effect has the potential to be more important than the first, given the strong radial dependence of the excitation field strength already discussed. It is our opinion that these geometrical effects are the dominant resolu- 
tion-limiting effects for high mass analyses in commercial instruments. A full accounting of these geometrical effects is beyond the scope of this article, but it is clear that both effects may be mitigated by use of small apertures, which limit ion detection to those trajectories near the axis of symmetry.

\section{Implications for High Mass Analyses}

The driving force behind examinations of ion trap resolution in mass-selective instability scans has been attempts to obtain high resolution mass spectra at very high mass-to-charge ratios $[10,29]$. Because a given instrument is generally limited to a maximum operating voltage either by electrical discharge or engineering constraints, three means have been used to extend the mass range of the ion trap to allow ejection of high mass ions: reduction of the resonance ejection operating point $q_{z}[5,30]$, reduction of the operating frequency $\Omega[31,32]$, and reduction of the trap radius $r_{\mathrm{o}}$ $[31,32]$. When one or more of these means is coupled with slow scan speeds, a combination of high mass analysis and high resolution may be obtained.

Use of any one of these three means to expand the mass range results in temperature-limited resolution because $k T \rightarrow C^{*} M r_{0}^{2} \omega^{2} / 6$. In the case of this limit, eq 32 may be rewritten as

$$
\frac{m}{\Delta m}=\frac{\sqrt{\varepsilon_{0}} r_{0}\left(q_{2} \Omega\right)^{2} m^{3 / 2}}{16 \pi n e \sqrt{2 \alpha_{\mathrm{p}} M k T}} \quad \sqrt{a} \tau \leqslant 1, k T>\frac{C^{*}}{6} M r_{\mathrm{o}}^{2} \omega^{2}
$$

which is the primary resolution expression for high mass analyses.

The $m^{3 / 2}$ dependence in eq 43 indicates promising resolution possibilities, but is in itself misleading. If the operating voltage could increase without limit, this would describe the mass dependence of resolution. In practice, the highest mass-to-charge ratio detectable is directly proportional to the maximum operating voltage according to the definition of $q_{z}$ in eq 2 . Substitution of the $q_{z}$ expression into eq 43 allows expected resolution at high masses to be written as

$$
\begin{aligned}
& \frac{m}{\Delta m} \simeq \frac{\sqrt{\varepsilon_{\mathrm{o}}} e V^{2}}{\pi n r_{\mathrm{o}}^{3} \Omega^{2} \sqrt{2 \alpha_{\mathrm{p}} m M k T}} \\
& \sqrt{a} \tau \ll 1, k T \lesssim \frac{C^{*}}{6} M r_{\mathrm{o}}^{2} \omega^{2}
\end{aligned}
$$

Equation 44 clarifies that once the maximum value of $V$ has been reached, resolution as expected decreases with increasing mass because the operating point $q_{z}$ must be reduced to extend the mass range of the instrument.

It is not clear that reductions in $r_{\mathrm{o}}$ or $\Omega$ in eq 44 are accompanied by an increase in the operating point and that this procedure may only continue until a maximum effective operating point for the trap is reached. This maximum effective value from other experiments appears to be $q_{z}=0.73[10,18]$ rather than the expected stability limit of $q_{z}=0.91$.

A clear impression of the maximum possible resolution at high mass may be obtained by a different substitution of $q_{z}$ into eq 43 . Substituting for the product $r_{\mathrm{o}} \Omega^{2}$, we obtain

$$
\frac{m}{\Delta m} \simeq \frac{\sqrt{\varepsilon_{\mathrm{o}} m} q_{z}\left(V / r_{\mathrm{o}}\right)}{4 \pi n \sqrt{2 \alpha_{\mathrm{p}} M k T}} \quad \sqrt{a} \tau \ll 1, k T>\frac{C^{*}}{6} M r_{\mathrm{o}}^{2} \omega^{2}
$$

which describes the maximum possible resolution for an ion of mass $m$ at operating point $q_{z}$. The ratio $V / r_{\text {o }}$ comes from electrical-discharge considerations and represents a maximum value that is related to a maximum electric field strength. It is also important to notice that this result is independent of the electrical charge of the ion.

It is important to recognize that this result is based on the temperature-limited regime, but that reductions in operating frequency actually increase the secular frequency $\omega$ so that the $k T \rightarrow C^{*} M r_{0}^{2} \omega^{2} / 6$ condition may be altered. It is also important to note that a similar evaluation of eq 33 to describe the maximum possible resolution for any ion is more difficult and beyond the scope of this paper.

The analysis that produced eqs 43 through 45 utilized the ion-induced dipole-interaction model. Once an ion is sufficiently large, the probability that a neutral molecule will interact with the charge center rather than a neutral portion of the ion structure becomes increasingly small. The ion-neutral interaction radius is given by

$$
b_{\mathrm{o}}^{2}=\frac{2 e}{v_{\mathrm{r}}} \sqrt{\frac{\alpha_{\mathrm{p}}}{\varepsilon_{\mathrm{o}} M}} \quad m>M
$$

When the ion dimensions are on the order of $b_{0}$, we may expect that a hard-space-type scattering model will take over, so that the mass dependence of $c$, as per eq 11 , will relate to the molecular cross section of the ion (i.e., $\sigma_{D}$ is proportional to $\mathrm{m}^{2 / 3}$ ). Once this limit is reached, the analog on eq 43 becomes

$$
\frac{m}{\Delta m} \propto \frac{r_{\mathrm{o}}\left(q_{z} \Omega\right)^{2} m^{5 / 6}}{n \sqrt{k T}} \quad \sqrt{a} \tau \ll 1, k T \gg \frac{C^{*}}{6} M r_{\mathrm{o}}^{2} \omega^{2}
$$

for very large $m$. Similar analogies to eqs 44 and 45 indicate that resolution decreases with increasing mass (once the voltage limit is reached) by factors of $m^{-7 / 6}$ and $m^{-1 / 6}$, respectively.

Ultimately the improvement of resolution will cease to keep up with the increasing mass. By using values 
for the polarizability of $\mathrm{N}_{2}$ and He included in Mason [13], we can show that the collision radius $b_{\mathrm{o}}$ is $12.5 \AA$ for $\mathrm{N}_{2}$ and $7.3 \AA$ for He with respect to a singly charged ion. These values are easily exceeded by the dimensions of a singly charged ion at $10,000 \mathrm{u}$, so that expected resolutions for such high mass ions will require extremely low pressures to compensate for the less favorable hard-sphere-type interaction conditions.

\section{Beyond the Pseudopotential-Well Approximation}

At several times during this discussion, we alluded to behavior of resonance ejection scans for $q_{z}$ values beyond the 0.4 limit of the pseudopotential-well approximation. This is of course because the resonance phenomena themselves are still active at these higher $q_{z}$ values $[8,9]$. Furthermore, we believe there is nothing conceptually flawed about the use of this description at higher $q_{z}$ values if appropriate considerations are developed. It is for this reason that we have typically utilized $\omega$ rather than $q_{z} \Omega$ in resolution (and other) expressions so that they may be extended to high $q_{z}$ values.

The first consideration that must be addressed to extend the present work beyond the pseudopotentialwell region is the use of a harmonic oscillation model to determine the amplitude dispersion in eq 14. Outside the pseudopotential region, $v_{\text {rms }}$ as used in eq 13 is no longer accurate because harmonic components of the oscillation make important contributions to the rms velocity. Therefore $\triangle A$ as developed in eq 14 is typically too small at high $q_{z}$ values. This should be compared to the results of Louris et al. [19], which indicate resolution decreases for $q_{z}$ values above 0.73 .

Second, the use of eq 1 implies a more basic assumption; this is, the amplitude distribution is unchanged during the scanning of the mass peak. At high scan speeds (i.e., $a^{1 / 2} \tau>1$ ) this is clearly true, but at low scan speeds, additional questions are raised. In effect, ions may "diffuse" from low to high amplitude regions of the distribution because of collisions with neutrals. This diffusion occurs at a rate that is proportional to the reduced collision frequency times the width of the distribution. Therefore as long as eq 48 is obeyed,

$$
\frac{d A}{d t} \gg c \Delta A
$$

the distribution will remain largely unchanged during scanning. It is interesting to note that by substitution this expression can be shown to be equivalent to the condition $a^{1 / 2} \tau \neq 1$.

At low-scan speeds, this implies that the ions may actually "diffuse" out of the trap prior to the normal ejection point of the distribution. In other words, most ions will enter a sufficiently high trajectory for ejection prior to the time when the bulk of the unaltered distribution would have reached the edge of the trap aperture. This effect may alter the ejection time for ions, but it does not alter the peak width because the relaxation time $\tau$ is proportional to $\Delta A /(\delta A / \delta t)$ in the pseudopotential-well region and in a diffusive ejection process, the peak width is also proportional to this relaxation time.

At high $q_{z}$ values the rate at which ions may change amplitude trajectories is altered by a secondary effect. 'The oscillation amplitude now is based on a superposition of multiple frequencies. Without collisions the phase relationship between the secular and trapping frequencies is determined by the voltages driving the oscillations and the initial conditions, but the presence of collisions may alter the phase relationship between the two oscillation frequencies. If the different oscillations have amplitudes on the same order of magnitude, it is clear that minor changes in phase due to collisions may produce alterations in amplitude on the order of $\Delta A$ from a single collision. In other words, the collision frequency of the ions with individual neutrals may determine the rate at which ions diffuse out of the trap at lower scan speeds. Therefore the actual collision frequency $c^{\prime}$ described by

$$
c^{\prime}=n v_{\mathrm{r}} \sigma_{\mathrm{p}}=\frac{m+M}{M} c
$$

determines the relaxation time constant $\tau^{\prime}$, and the temporal line width $\Delta t$ may be expressed as the smaller of eq 20 and $\tau^{\prime} \equiv 4 c^{\prime}$.

These additional caveats are added to the previous geometrical considerations. Figure 6 illustrates a comparison between theoretical predictions and recent experimental results published by the Finnigan group (Louris et al. [19]) and by Loundry et al. [3]. Both sets of results illustrate resolutions between 2 and 4 orders of magnitude greater than the frequency line width model predicts. Figure 6a compares the maximum resolution prediction of the present model to values obtained by Louris et al. [19] for the $\mathrm{m} / \mathrm{z} 129$ ion of xenon ejected at $q_{z}=0.73$. It already has been noted that a voltage prediction of $4.2 \mathrm{~V}$ to obtain maximum resolution agrees very well with the value of $\sim 5 \mathrm{~V}$ obtained by Louris et al. [19] at a scan rate of $5500 \mathrm{u} / \mathrm{s}$. It should be noted that eq 41 predicts that maximum resolution will be obtained at $V_{\mathrm{s}}$ values beyond the capability of the commercial instrument for higher mass ions $(m / z>100)$. Resolution improvements for high mass ions produced by using axial excitation voltages between 6 and $25 \mathrm{~V}$ were recently demonstrated in our laboratory [26].

Figure $6 \mathrm{~b}$ compares predictions of the present model with results recently reported by Loundry et al. [3]. The lack of $a^{1 / 2}$ dependence ( $w$ hich is indicated by the solid line), the lack of mass dependence, and the fact that that present theoretical construction still underestimates the resolution line by a factor of 10 are of considerable concern. The authors believe this is due to 

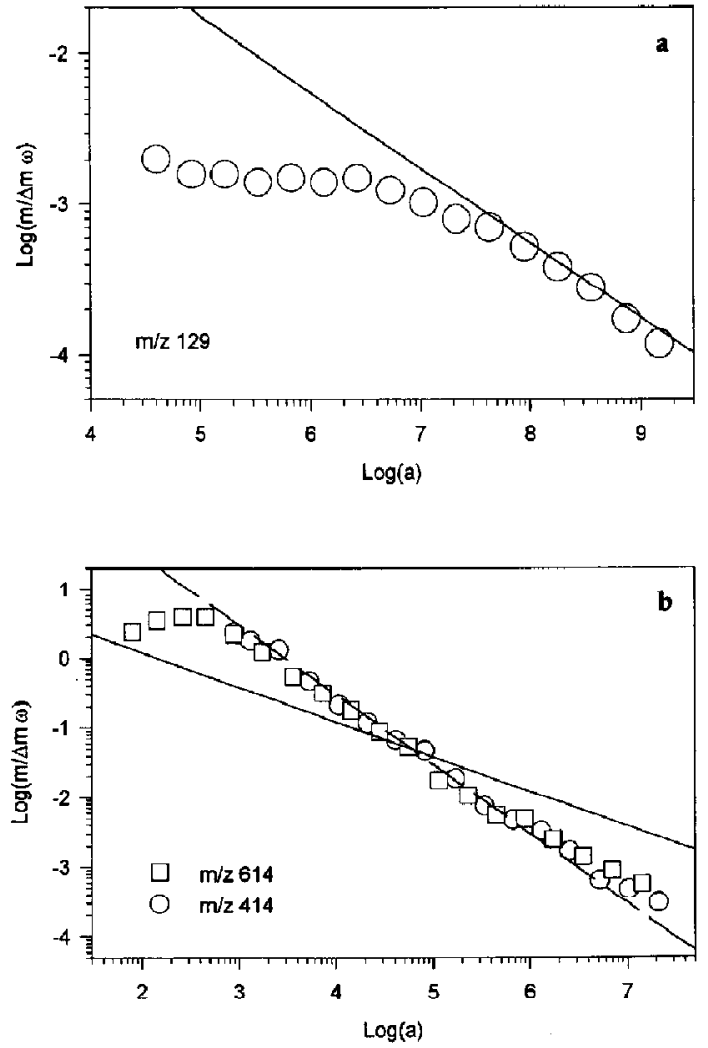

Figure 6. Comparison of the present model with results obtained by (a) Louris et al. (19) and by (b) Loundry et al. (3). The solid line indicates predicted maximum resolution. Additional details are in the text.

a collection of the previously mentioned secondary effects. The dotted line in this figure corresponds to

$$
\frac{m}{\Delta m}=\frac{\omega c^{\prime}}{2 a}
$$

This is the expected scan-rate dependence when the temporal line width is determined by the collision relaxation time $\tau^{\prime}$. In this case, we took the pressure to be $10^{-4}$ torr and temperature to be $300 \mathrm{~K}$. The highest resolution is obtained at $a^{1 / 2} t \simeq 1$ in this curve, and the peak width is determined by the $\tau^{\prime}$ until eq 20 again becomes the limiting expression for line width. At the higher scan speeds, the $a^{1 / 2}$ dependence returns, but the resolution is less than predicted because of (at least) two factors we have already mentioned: (1) the geometrical considerations in aperture shape and dipole excitation and (2) limitation of excitation amplitudes to $6 \mathrm{~V}$ on commercial instruments. Two reasons for not observing this same phenomenon in Figure 6a also should be noted: (1) the lower $q_{z}$ value in the Louris et al. [19] data reduces the drive frequency amplitude with respect to secular amplitude and (2) the initial amplitude dispersion is wider for the lower mass jons.

\section{Conclusion}

The present amplitude-dispersion-based additions to the frequency line-width-based model of Goeringer et al. [10] offer considerable insight into the phenomena that affect resolution in ion-trap-based instruments. Furthermore, it appears to do so at high scan speeds and $q_{z}$ values that are beyond the limiting cases used in the construction of the theory, even though considerable effort may be required to develop the appropriate correction values required to make the theory quantitative for predictions in these regions. Ongoing efforts by other researchers in the areas of temperature estimation [21-23], nonlinear resonances $[9,24]$, and ion trajectory studies $[33,34]$ as well as additional efforts in the areas of geometrical issues $[27,28]$ and collisional effects $[33,34]$ on ion packet trajectories will all contribute to the ultimate understanding of ion trap resolution properties.

\section{Acknowledgments}

This work was sponsored by the Army Research Office under contract DAAL03-91-G-0296.

\section{References}

1. Paul, W.; Reinhard, H. P.; Von Zahn, U. Z. Phys. 1958, 152, 143.

2. Williams, J. D.; Cux, K.; Murand, K. L.; Couks, R. G.; Julian, R. K.; Kaiser, R. E. In Proceddings of the 39 th ASMS Conference on Mass Spectrometry and Allied Topics, Nashville, 1991; $\mathrm{p}$ 1481.

3. Loundry, F. A.; Wells, G. J.; March, R. E. Rapid Commun. Mass Spectrom. 1993, 7, 43.

4. Kaiser, R. E., Jr.; Cooks, R. G.; Stafford, G. C.; Syka, J. E. P.; Hemberger, P. H. Int. J. Mass Spectrom. Ion Process. 1991, 106 , 79 .

5. Kaiser, R. E.; Louris, J. N.; Amy, J. W.; Cooks, R. G. Rapid Commun. Mass Spectrom. 1989, 3, 225.

6. Kelley, P. E.; Stafford, G. C.; Stephens, D. R. U.S. Patent 4,540,884, September 10, 1985.

7. March, R. E.; Hughes, R. J. In Quadrupole Storage Mass Spectrometry; Winefordner, J. D.; Kolthoff, I. M., Eds.; Chemical Analysis Series 102; Wiley: New York, 1989.

8 Syka, J. E. P.; Louris, J. N.; Kelley, P. E.; Stafford, G. C.; Reynolds, W. E. U.S. Patent 4,736,101, 1988.

9. Franzen, J.; Gabling, R.-H.; Heinen, G.; Weiss, G. U.S. Patent 4,882,484, November 21, 1989.

10. Goeringer, D. E.; Whitten, W. B.; Ramsey, J. M.; McLuckey, S. A.; Glish, G. L. Anal. Chem. 1992, 64, 1434.

11. Major, F. G.; Dehmelt, H. G. Phys. Rev. 1968, 170, 91-107.

12. Marion, J. B. Classifcal Dynamics of Particles and Systems; Academic Press: New York, 1970; p 117.

13. Mason, E. A.; McDaniel, E. W. Transport Properties of Ions in Gases; Wiley Interscience: New York, 1988; p 144,

14. Gautschi, W. In Handbook of Mathematical Functions; Abramowitz, M,; Stegun, I. A., Eds.; Dover: New York, 1972; pp 295-330.

15. Fowles, G. R. Introduction to Modern Optics; Holt, Rinehart, and Winston: New York, 1975; pp 125-135. 
16. Stafford, G. C.; Kelley, P. E.; Syka, J. E. P.; Reynolds, W. E.; Todd, J. F. J. Int. J. Mass Spectrom. Ion Process. 1984, 60, 85.

17. Weber-Grabat1, M.; Kelley, P. E.; Bradshaw, S. C.; Hockmann, D. J. In Proceedings of the 36th ASMS Conference or Mass Spectrometry and Allied Topics, San Francisco, 1988; p 1106.

18. Schwarz, J. C.; Syka, J. E. P.; Jardine, I. J. Am. Soc. Mass Spectrom. 1991, 2, 198.

19. Louris, J.; Freuler, S.; Kirkish, J.; Schwartz, J.; Statford, G.; Syka, J.; Taylor, D.; Tucker, D.; Zhun, J. In Proceedings of the 39th ASMS Conference on Mass Spectrometry and Allied Topics, Nashville, TN, 1991; p 542.

20. Cameron, D.; Hemberger, P. H.; Alarid, J. E.; Liebman, C. P.; Williams, J. D. J. Am. Soc. Mass Spectro. 1993, 4, 774.

21. Vedel, F. Int. J. Mass Spectrom. Ion Process. 1991, 106, 33.

22. Wu, H.-F.; Brodbelt, J. S. Int. J. Mass Spectrom. Ion Process. 1992, 115, 617

23. Basic, C.; Eyles, J. R.; Yost, R. A. J. Am. Soc. Mass Spectrom. 1992, 3, 716 .

24. Vedel, F; Vedel, M.; March, R. E. Int. J. Mass Spectrom. Ion Process. 1990, 99, 125.
25. McLuckey, S. A.; Goeringer, D. E.; Glish, G. L. Anal. Chem. $1992,64,1455$

26. Arnold, N. S.; McClennen, W. H.; Meuzclaar, H. L. C. In Proceedings of the 41st ASMS Conference on Mass Spectrometry and Allied Topics, San Francisco, CA, 1993; p 21a.

27. Philip, M. A.; Gelbard, F.; Amold, S. J. J. Colloid Interface Sci. 1980, 75, 566.

28. Davis, E. J. Langmuir 1985, 1, 379.

29. Cooks, R. G.; Amy, J.; Bier, M.; Schwartz, J.; Schey, K. Adv. Mass Spectrom. 1989, 11A, 33.

30. Fulford, J. E.; Hoa, D.-N.; Hughes, R. J.; March, R. E.; Bonner, R. F.; Wong, G. L. J. Vac. Sci. Technol. 1980, 17, 829.

31. Kaiser, R. E.; Cooks, R. G. Rapid Commun. Mass Spectrom. $1989,3,50$

32. Kaiser, R. E.; Cooks, R. G.; Moss, J.; Hemberger, P. H. Rapid Commun. Mass Spectrom. 1989, 3, 50.

33. Williams, J. D.; Cooks, R. G.; Syka, J. E. P.; Hemberger, P. H.; Nogar, N. S. J. Am. Soc. Mass Spectrom. 1993, 4, 792.

34. Loundry, F. A.; Alfred, R. L; March, R. E. I. Am. Soc. Mass Spectrom. 1993, 4, 687 . 